CTNI-62 CLINICAL FEATURES AND PROGNOSTIC FACTORS OF NONGERMINOMATOUS GERM CELL TUMORS IN 111 CONSECUTIVE PATIENTS IN A SINGLE INSTITUTION: IMPACT OF IRRADIATION AND CHEMOTHERAPY CYCLES ON SURVIVAL Lei Wen, Juan Li, Qingjun Hu, Mingyao Lai, Cheng Zhou, Changguo Shan, and Linbo Cai; Guangdong Sanjiu Brain Hospital, Guangzhou, China (People's Republic)

BACKGROUND: Limited data is available in intracrania nongerminomatous germ cell tumors (NGGCTs) in Chinese population. Here we aimed to retrospectively assess the clinical-pathological and prognostic factors of NGGCTs in a single large institution in China. METHODS: From June 2003 to December 2018, 111 consecutive NGGCTs were treated in Guangdong Sanjiu Brain Hospital, China. RESULTS: The median follow-up was 36.2 months (range, 1.2 to 131.2 months). Three-year EFS and OS for 111 NGGCTs patients were $78.5 \% \pm 4.5 \%$ and $82.8 \% \pm 4.0 \%$, respectively 98 patients received CSI plus boost yielded better survival than those who received reduced-volume radiotherapy or no radiotherapy (3y OS, 86.7\% vs. $51.4 \%, p=0.007)$. Patients had at least four cycles of chemotherapy were strongly associated with improved 3-year OS, compared to those received less than 4 cycles $(94.1 \%$ vs. $63.6 \%, \mathrm{p}<0.001)$. There was no significant difference in survival of patients stratified by age, surgery, hydrocephalus, as well as tumor diameter. Multivariate analysis identified chemotherapy cycles less than 4 was the only prognostic factor that conferring a worse OS $(p=0.003)$. Patients both received CSI and at least 4 courses of chemotherapy were correlated with lower incidence of relapse $(\mathrm{p}=0.044)$. CONCLUSIONS: Multimodal approach including CSI and enough courses of chemotherapy was effective and should be recommended for the treatment of newly diagnosed NGGCTs in Chinese population.

CTNI-63. PROGNOSTIC FACTORS IN PATIENTS WITH BASAL GANGLIA GERM-CELL TUMORS: A RETROSPECTIVE ANALYSIS OF THE SINGLE CHINESE INSTITUTE EXPERIENCE FROM 2009 TO 2019

Qingjun Hu, Juan Li, Mingyao Lai, Cheng Zhou, Zhaoming Zhou, Changguo Shan, and Linbo Cai; ${ }^{1}$ Guangdong Sanjiu Brain Hospital, Guangzhou, China (People's Republic)

OBJECTIVE: To evaluate the clinical factors related to the prognosis of basal ganglia germ cell tumors. METHODS: A retrospective analysis of 52 cases of the basal ganglia germ cell tumors treated from January 2009 to January 2019 in the department of oncology of Guangdong Sanjiu Brain Hospital. The median age: 12 years (range: 5-32), The median course of disease: 11.7 months (range: 1-54). Thirteen cases were diagnosed by biopsy and 39 cases were diagnosed by elevated tumor markers. There wer 31 patients $(59.6 \%)$ diagnosed with germinomas and 21 patients $(40.4 \%$ with non-germ germ cell tumors. Univariate and multivariate survival analysis was performed. RESULTS: To October 15, 2019, the median follow-up time was 30.4 months (range $2-124$ months). The 5 -year survival rate was $85 \%$, and the 5 -year progression-free survival rate was $84 \%$. Multivariate analysis found whether serum AFP was greater than $100 \mathrm{mIU} / \mathrm{ml}$, (with $\mathrm{HR}$ : 11.441,95\% CI: 2.09-47.66, P = 0.005), the degree of surgical resection(with HR 5.323 (1.19-23.812), P =0.029), PD as the effect of radiotherapy (HR $16.53,(1.19-23.81), \mathrm{P}=0.001)$ were independent prognostic factor af fecting survival. CONCLUSION: The pathological type, degree of surgical resection, and response to initial treatment can all affect survival.

\section{CTNI-64. MATRIX REGIMEN FOR NEWLY DIAGNOSED PRIMARY DIFFUSE B-CELL LYMPHOMA OF THE CENTRAL NERVOUS} SYSTEM

Richard Green, and Colleen Thornton; Kaiser Permanente-Los Angeles Medical Center, Los Angeles, CA, USA

We report our experience treating 16 patients with newly diagnosed primary diffuse B-cell lymphoma of the central nervous system with the MATRix regimen as reported by Ferreri, et al in 2016. The median age was 66 years $(y) ; 8$ patients were men and 8 were women. Fourteen had cerebral mass lesions, 1 had a paraspinal mass, and 1 leptomeningeal disease. Treatment consisted of 4 cycles of rituximab $375 \mathrm{mg} / \mathrm{sq} \mathrm{m}$ (Days 1, 6); methotrexate $3.5 \mathrm{~g} / \mathrm{sq} \mathrm{m}$ (Day 2); cytarabine $2 \mathrm{~g} / \mathrm{sq}$ m every $12 \mathrm{~h}$ (Days 3, 4); and thiotepa $30 \mathrm{mg} / \mathrm{sq} \mathrm{m}$ (Day 5). Patients without disease progression received carmustine $400 \mathrm{mg} / \mathrm{sq} \mathrm{m}$ and thiotepa $4 \times 5 \mathrm{mg} / \mathrm{kg}$ followed by autologous bone marrow transplantation. Four patients were switched to MATRix after 6 cycles of methotrexate and rituximab and received only 2 initial cycles including cytarabine and thiotepa. Overall, 12 patients completed initial chemotherapy. Eleven of these 12 went on to transplantation; one patien had early disease progression and did not proceed to transplantation. All 4 patients over 70 y died early after 1-2 cycles; the median age of these was $80 \mathrm{y}$, and the causes of early death were neutropenic fever, septic thrombophlebitis, and cardiac arrest. One patient who completed the entire regimen including transplantation died of fungal pneumonia; the other 11 experi- enced no serious acute toxicity. One patient had late disease progression; one developed symptomatic leukoencephalopathy. Nine of the 16 patients remain alive. At a median follow-up of 23 months, median time to progression was 421 days and overall survival 564 days. These preliminary data suggest that the MATRix regimen in safe and highly effective in the newly diagnosed setting. However, the high incidence of early death in patients over 70 y suggests that the regimen may be too toxic for elderly individuals.

\section{CTNI-65. COMBINED RITUXIMAB, METHOTREXATE,} PROCARBAZINE, VINCRISTINE, AND CYTARABINE (R-MPV-A) TREATMENT FOR PATIENTS WITH RELAPSED PRIMARY CNS LYMPHOMA

Motoo Nagane $^{1}$, Nobuyoshi Sasaki ${ }^{1}$, Keiichi Kobayashi ${ }^{1}$, Kuniaki Saito ${ }^{1}$, Daisuke Shimada $^{1}$, Yoshie Matsumoto ${ }^{1}$, Shohei Iijima ${ }^{1}$, Yuki Yamagishi ${ }^{1}$, Saki Shimizu ${ }^{1}$, Nobuyuki Takayama ${ }^{2}$, and Yoshiaki Shiokawa ${ }^{1}$;

${ }^{1}$ Department of Neurosurgery, Kyorin University Faculty of Medicine, Tokyo, Japan, ${ }^{2}$ Department of Hematology, Kyorin University Faculty of Medicine, Tokyo, Japan

BACKGROUNDS: Standard of care for patients with primary CNS lymphoma (PCNSL) has been high-dose methotrexate (HD-MTX)-based multiagent immunochemotherapy including an induction R-MPV with consolidation whole-brain radiotherapy (WBRT) and HD-cytarabine to achieve complete remission, however, the optimal treatment for relapsed/refractory $(\mathrm{r} / \mathrm{r})$ PCNSL has not been established yet, besides the recent approval of BTK inhibitor, tirabrutinib, in Japan. We evaluated retrospectively efficacy of R-MPV-A for r/rPCNSL. PATIENTS: Histologically proven PCNSL patients treated at relapse in our institution from April 2000 to November 2019 were analyzed. Outcomes were compared between those treated with RMPVA or other regimens. RESULTS: Among 148 PCNSL patients identified, 73 had at least one relapse, of whom 47 received salvage chemotherapy including 23 treated with RMPVA either at the first relapse (11) or at the second or later relapse (12). Median age/KPS of this salvage RMPVA group were $71(42-87) / 80(40-100)$. Nine patients were pretreated with the firstline RMPVA. HD-cytarabine was given to 13 patients. Median number of RMPV cycles was 8 (1-4 cycles: $10 ; 8$ cycles 13$)$. CR/CRu were achieved in $19(83 \%)$, response rate was $87 \%$, while there were two PDs $(9 \%)$. After median follow-up of 21.9 months, the median PFS after salvage RMPVA was 13.0 m (95\% CI: 9.1-16.9), 1-year overall survival (OS) was $82 \%$, median OS was $70.0 \mathrm{~m}$ (95\%CI: 12.9-127.1), which were longer than those in 24 patients with salvage treatment other than RMPVA (mPFS $4.4 \mathrm{~m}$ $\mathrm{P}=0.054$; mOS $13.6 \mathrm{~m}, \mathrm{P}=0.009$ ). Among patients with salvage RMPVA, HD-cytarabine was associated with better PFS $(\mathrm{P}=0.066)$, and complete responses were significantly correlated with longer OS. Treatment was generally well-tolerated but there was one treatment-related death. CONCLUSIONS: Salvage RMPVA at relapses was active and associated with longer survival compared with other regimens, necessitating further development of salvage regimens incorporating tirabrutinib in the future studies.

CTNI-66. ONE-YEAR FOLLOW-UP DATA OF PHASE I/II STUDY OF TIRABRUTINIB IN PATIENTS WITH RELAPSED OR REFRACTORY PRIMARY CENTRAL NERVOUS SYSTEM LYMPHOMA

Kazuhiko Mishima ${ }^{1}$, Yoshitaka Narita ${ }^{2}$, Motoo Nagane ${ }^{3}$,

Yasuhito Terui ${ }^{4}$, Yoshiki Arakawa ${ }^{5}$, Hajime Yonezawa ${ }^{6}$, Katsunori Asai ${ }^{7}$, Noriko Fukuhara $^{8}$, Kazuhiko Sugiyama ${ }^{9}$, Naoki Shinojima ${ }^{10}$, Arata Aoi ${ }^{11}$, and Ryo Nishikawa ${ }^{1} ;{ }^{1}$ Department of Neuro-Oncology/Neurosurgery, Saitama Medical University International Medical Center, Saitama, Japan, ${ }^{2}$ Department of Neurosurgery and Neuro-Oncology, National Cancer Center Hospital, Tokyo, Japan, ${ }^{3}$ Department of Neurosurgery, Kyorin University Faculty of Medicine, Tokyo, Japan, ${ }^{4}$ Department of Hematology and Oncology, The Cancer Institute Hospital, Japanese Foundation for Cancer Research, Tokyo, Japan, ${ }^{5}$ Department of Neurosurgery, Kyoto University Graduate School of Medicine, Kyoto, Japan, ${ }^{6}$ Department of Neurosurgery, Kagoshima University Hospital, Kagoshima, Japan, ${ }^{7}$ Department of Neurosurgery, Osaka International Cancer Institute, Osaka, Japan, ${ }^{8}$ Department of Hematology and Rheumatology, Tohoku University Graduate School of Medicine, Sendai, Japan, ${ }^{9}$ Department of Medical Oncology, Hiroshima University Hospital, Hiroshima, Japan, ${ }^{10}$ Department of Neurosurgery, Kumamoto University Hospital, Kumamoto, Japan, ${ }^{11}$ Department of Clinical Development, Ono Pharmaceutical Co., LTD., Osaka, Japan

Primary central nervous system lymphoma (PCNSL) is known as one of the incurable brain tumors. In March 2020, Tirabrutinib (TIR), a secondgeneration oral Bruton's tyrosine kinase inhibitor, was approved for the indication of relapsed or refractory PCNSL (rrPCNSL) based on the results of a phase I/II study in Japan (Trial registration: JapicCTI-173646). In this study, 44 Japanese patients with rrPCNSL were treated with TIR QD at $320 \mathrm{mg}$, $480 \mathrm{mg}$, or $480 \mathrm{mg}$ in the fasted condition (480 $\mathrm{mg}$ fasted QD). The primary endpoint was overall response rate (ORR) assessed by an independent review committee according to International PCNSL Collaborative Group 